\section{Augmented endoscopic resection for fibrotic or recurrent colonic polyps using an ablation and cold avulsion technique}

Severe submucosal tethering can occur at the base of a polyp because of previous failed endoscopic attempts, extensive biopsies, de-novo fibrosis of flat polyps, or tattoo ink being placed inadvertently at the base of the polyp. A scarred submucosa limits the depth and effectiveness of the lifting solution used when resecting a polyp, which makes snare capture of fibrotic polyp segments impossible and risks the resection being incomplete [ 1 , 2]. Japanese authors have suggested endoscopic submucosal dissection (ESD) en bloc resection of fibrotic polyps; however this is technically difficult and carries a higher risk of perforation $[3,4]$. Supplementary ablative techniques such as the use of either argon plasma coagulation (APC) with prior submucosal injection or hot avulsion using electrocautery biopsy forceps are alternative, simpler, and lower risk strategies $[2,5]$. The depth of tissue destruction is, however, difficult to accu- rately judge and viable polyp tissue remains below the cauterized surface when thermal energy alone is used.

We report our preliminary experience of a new salvage approach to achieve complete eradication of partially lifting or nonlifting, benign, fibrotic polyps using an ablation and cold avulsion (ACA) technique. After submucosal injection had been performed, a conventional piecemeal snare "lift and cut" endoscopic mucosal resection (EMR) was performed on all polyp tissue where lifting was adequate. Residual scarred tissue was initially ablated using high power APC (ERBE-VIO, 25-40W flow, 1.6-2 L/min; Erbe, Tübingen, Germany) and this was followed by "cleaning" of the cauterized polyp tissue using a nonspiked biopsy forceps. Repeat APC application and polyp cleaning were performed until the submucosal scar tissue was visible ( $\bullet$ Fig. 1 ; $\bullet$ Video 1 ).
The ACA rescue technique was applied successfully (after consent had been obtained from the patients) to 15 consecutive fibrotic polyps after piecemeal EMR polypectomy. An apparent complete polypectomy was achieved in all cases ( $\bullet$ Table 1). The avulsed specimens revealed low grade dysplastic tissue, verifying the tissue destruction by APC. Followup of 3-7 months showed residual polyp tissue ( $2 \mathrm{~mm}$ and $5 \mathrm{~mm}$ ) in 2 of the 14 patients assessed to date, which was suc-

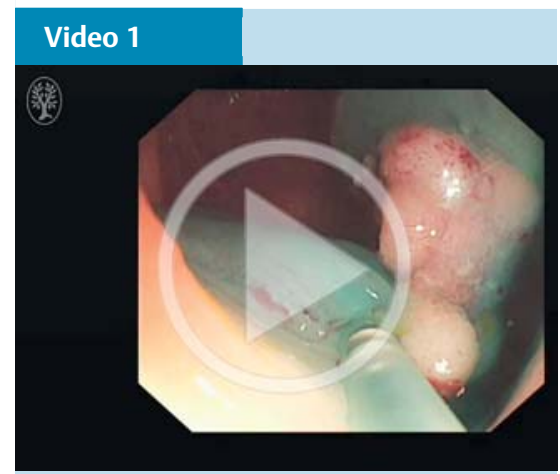

A recurrent fibrotic polyp being treated with piecemeal endoscopic mucosal resection (EMR) followed by the ablation and cold avulsion (ACA) technique.
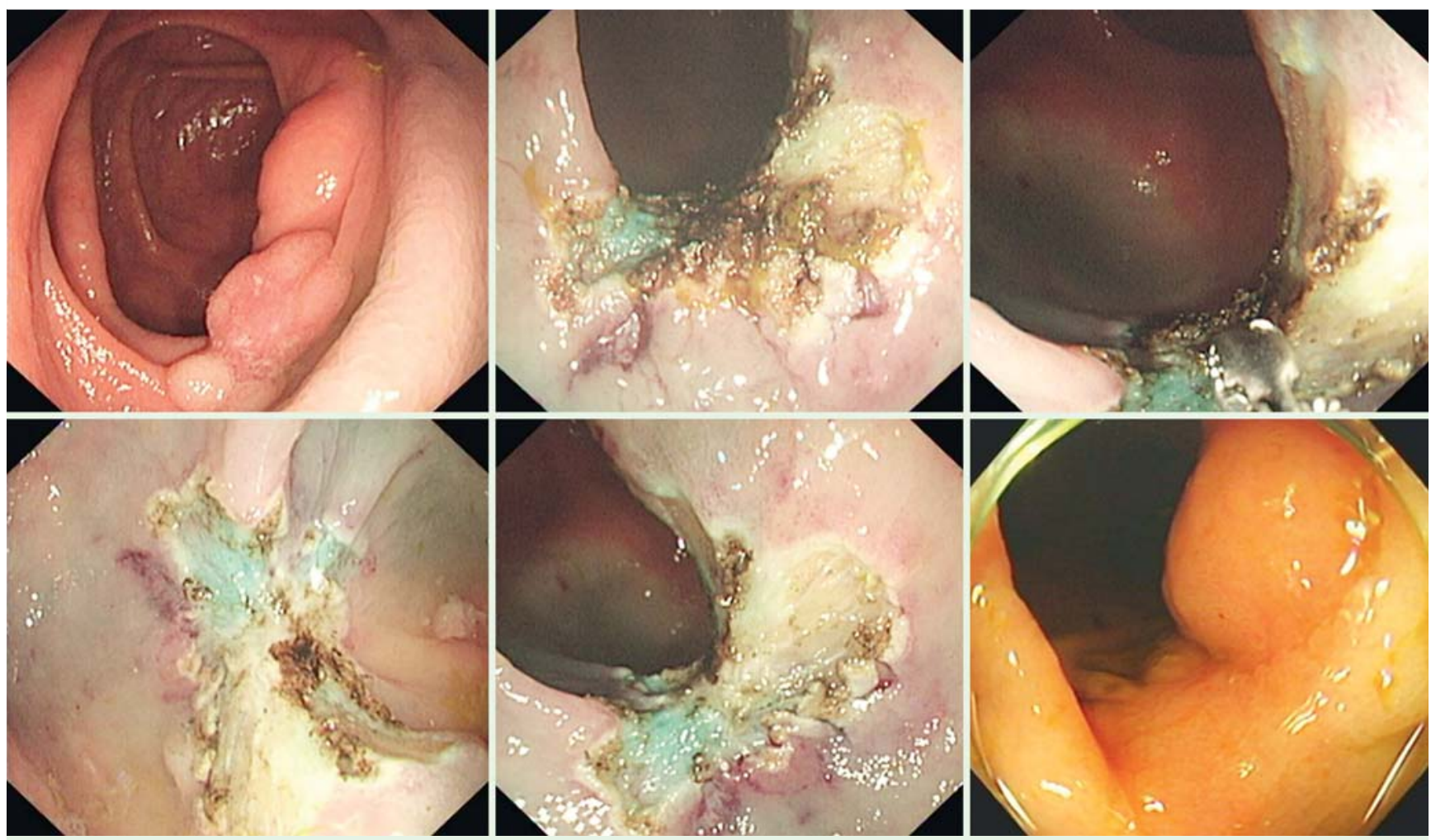

Fig. 1 Endoscopic images from patient \#5, an 81-year-old man with a recurrent proximal ascending colon polyp, showing: a a 2.5-cm recurrent fibrotic adenoma; $\mathbf{b}$ the fibrotic base after ablation; $\mathbf{c}$ avulsion with the cold biopsy forceps; $\mathbf{d}$ the scarred base after avulsion; e the final resection defect; $\mathbf{f}$ the healed scar that is free of recurrence at follow-up. 
Table 1 Characteristics of 15 patients with fibrotic polyps that were treated using the ablation and cold avulsion technique, and clinical outcome of the procedure.

\begin{tabular}{|c|c|c|c|c|c|c|c|c|c|}
\hline $\begin{array}{l}\text { Patient } \\
\text { number }\end{array}$ & Age; sex & Site & $\begin{array}{l}\text { Size, } \\
\mathrm{cm}\end{array}$ & $\begin{array}{l}\text { De novo/ } \\
\text { recurrent } \\
\text { polyp }\end{array}$ & $\begin{array}{l}\text { Morphologic } \\
\text { type }\end{array}$ & $\begin{array}{l}\text { Previous } \\
\text { resection } \\
\text { attempts }\end{array}$ & Histology & $\begin{array}{l}\text { Follow-up, } \\
\text { months }\end{array}$ & Outcome \\
\hline 1 & 74; male & $\begin{array}{l}\text { Ascending } \\
\text { colon }\end{array}$ & 1 & Recurrent & Ila & Yes & $\begin{array}{l}\text { Tubular adenoma + low } \\
\text { grade dysplasia }\end{array}$ & 5 & $\begin{array}{l}\text { No } \\
\text { recurrence }\end{array}$ \\
\hline 2 & 72; male & $\begin{array}{l}\text { Transverse } \\
\text { colon }\end{array}$ & 3 & Recurrent & $\mathrm{Ila}+\mathrm{Is}$ & Yes & $\begin{array}{l}\text { Tubulovillous adenoma } \\
+ \text { low grade dysplasia }\end{array}$ & 5 & $\begin{array}{l}\text { No } \\
\text { recurrence }\end{array}$ \\
\hline 3 & 58; male & $\begin{array}{l}\text { Sigmoid } \\
\text { colon }\end{array}$ & 0.5 & Recurrent & Is & Yes & $\begin{array}{l}\text { Tubulovillous adenoma } \\
+ \text { low grade dysplasia }\end{array}$ & 6 & $\begin{array}{l}\text { No } \\
\text { recurrence }\end{array}$ \\
\hline 4 & 79; male & $\begin{array}{l}\text { Transverse } \\
\text { colon }\end{array}$ & 3 & De novo & $\begin{array}{l}\text { Nongranular } \\
\text { LST (Ila) }\end{array}$ & No & $\begin{array}{l}\text { Tubular adenoma + low } \\
\text { grade dysplasia }\end{array}$ & 4 & $\begin{array}{l}\text { 2-mm } \\
\text { recurrence }\end{array}$ \\
\hline 5 & 81 ; male & $\begin{array}{l}\text { lleocecal } \\
\text { valve }\end{array}$ & 2.5 & Recurrent & Is & Yes & $\begin{array}{l}\text { Tubulovillous adenoma } \\
\text { + low grade dysplasia }\end{array}$ & 5 & $\begin{array}{l}\text { No } \\
\text { recurrence }\end{array}$ \\
\hline 6 & 73; female & $\begin{array}{l}\text { Transverse } \\
\text { colon }\end{array}$ & 5 & De novo & $\begin{array}{l}\text { Mixed LST } \\
(\mathrm{Il} a+\mathrm{Is})\end{array}$ & Yes & $\begin{array}{l}\text { Tubulovillous adenoma } \\
\text { + low grade dysplasia }\end{array}$ & 4 & $\begin{array}{l}\text { No } \\
\text { recurrence }\end{array}$ \\
\hline 7 & 72; male & $\begin{array}{l}\text { lleocecal } \\
\text { valve }\end{array}$ & 0.5 & Recurrent & Is & Yes & $\begin{array}{l}\text { Tubular adenoma + low } \\
\text { grade dysplasia }\end{array}$ & 6 & $\begin{array}{l}\text { No } \\
\text { recurrence }\end{array}$ \\
\hline 8 & 51 ; male & $\begin{array}{l}\text { Ascending } \\
\text { colon }\end{array}$ & 2 & Recurrent & $\mathrm{II} a+$ Is & Yes & $\begin{array}{l}\text { Tubulovillous adenoma } \\
\text { + low grade dysplasia }\end{array}$ & 4 & $\begin{array}{l}\text { No } \\
\text { recurrence }\end{array}$ \\
\hline 9 & 76; male & $\begin{array}{l}\text { Ascending } \\
\text { colon }\end{array}$ & 1.5 & Recurrent & Ila & Yes & $\begin{array}{l}\text { Tubulovillous adenoma } \\
\text { + low grade dysplasia }\end{array}$ & 7 & $\begin{array}{l}\text { No } \\
\text { recurrence }\end{array}$ \\
\hline 10 & 58; male & Cecum & 2 & De novo & Is & No & $\begin{array}{l}\text { Tubulovillous adenoma } \\
\text { + low grade dysplasia }\end{array}$ & 4 & $\begin{array}{l}\text { No } \\
\text { recurrence }\end{array}$ \\
\hline 11 & 61 ; female & Rectum & 1.6 & Recurrent & Ila & Yes & $\begin{array}{l}\text { Tubular adenoma + low } \\
\text { grade dysplasia }\end{array}$ & 5 & $\begin{array}{l}\text { No } \\
\text { recurrence }\end{array}$ \\
\hline 12 & 68; female & $\begin{array}{l}\text { Transverse } \\
\text { colon }\end{array}$ & 3 & De novo & $\begin{array}{l}\text { Granular LST } \\
(\mathrm{IIa}+\mathrm{Is})\end{array}$ & Yes & $\begin{array}{l}\text { Tubular adenoma + low } \\
\text { grade dysplasia }\end{array}$ & 5 & $\begin{array}{l}\text { 5-mm } \\
\text { recurrence }\end{array}$ \\
\hline 13 & 70; male & Rectum & 4 & Recurrent & $\begin{array}{l}\text { Nongranular } \\
\text { LST (Ila) }\end{array}$ & Yes & $\begin{array}{l}\text { Tubulovillous adenoma } \\
\text { + low grade dysplasia }\end{array}$ & $\begin{array}{l}\text { Awaiting } \\
\text { follow-up }\end{array}$ & - \\
\hline 14 & 65; female & $\begin{array}{l}\text { Recto } \\
\text { sigmoid }\end{array}$ & 3.5 & Recurrent & $\mathrm{IIa}+\mathrm{Is}$ & Yes & $\begin{array}{l}\text { Tubulovillous adenoma } \\
+ \text { low grade dysplasia }\end{array}$ & 3 & $\begin{array}{l}\text { No } \\
\text { recurrence }\end{array}$ \\
\hline 15 & 77; male & $\begin{array}{l}\text { Hepatic } \\
\text { flexure }\end{array}$ & 3 & De novo & Ila & No & $\begin{array}{l}\text { Tubulovillous adenoma } \\
\text { + low grade dysplasia }\end{array}$ & 3 & $\begin{array}{l}\text { No } \\
\text { recurrence }\end{array}$ \\
\hline
\end{tabular}

LST, laterally spreading tumor.

cessfully treated with further endoscopic therapy.

Our retrospective case series suggests that ACA is a safe and effective technique that could act as an adjunct to snare resection to achieve complete eradication of benign scarred polyps

Endoscopy_UCTN_Code_TTT_1AQ_2AD

\section{Competing interests: None}

\section{Zacharias P. Tsiamoulos ${ }^{1,}$ *, Rajaratnam Rameshshanker ${ }^{1, *}$, Sachin Gupta ${ }^{2}$, Brian P. Saunders ${ }^{1}$}

${ }^{1}$ Wolfson Unit for Endoscopy, St Mark's Hospital/Academic Institute, London UK

2 Department of Gastroenterology, Princess Alexandra Hospital, Harlow, UK

\footnotetext{
* contributed equally to this paper
}

\section{References}

1 Chedgy FJ, Bhattacharyya R, Kandiah $K$ et al. Knife-assisted snare resection: a novel technique for resection of scarred polyps in the colon. Endoscopy 2016; 48: 277-280

2 Tsiamoulos ZP, Bourikas LA, Saunders BP. Endoscopic mucosal ablation: a new argon plasma coagulation/injection technique to assist complete resection of recurrent, fibrotic colon polyps (with video). Gastrointest Endosc 2012; 75: $400-404$

3 Mizushima T, Kato M, Iwanaga I et al. Technical difficulty according to location, and risk factors for perforation, in endoscopic submucosal dissection of colorectal tumors. Surg Endosc 2015; 29: 133-139

4 Kim ES, Cho KB, Park KS et al. Factors predictive of perforation during endoscopic submucosal dissection for the treatment of colorectal tumors. Endoscopy 2011; 43: 573-578

5 Veerappan SG, Ormonde D, Yusoff IF et al. Hot avulsion: a modification of an existing technique for management of nonlifting areas of a polyp (with video). Gastrointest Endosc 2014; 80: 884-888

\section{Bibliography}

Dol http://dx.doi.org/

10.1055/s-0042-109601

Endoscopy 2016; 48: E248-E249

(c) Georg Thieme Verlag KG

Stuttgart · New York

ISSN 0013-726X

\section{Corresponding author}

\section{Zacharias P. Tsiamoulos, MBBS}

Wolfson Unit for Endoscopy,

Imperial College

St Mark's Hospital/Academic Institute

Watford Road

Harrow

Middlesex

HA1 3UJ

London

UK

ztsiamoulos@nhs.net 